\title{
Ordered Solution Generation for Implicit AND/OR Search Spaces
}

\author{
Priyankar Ghosh, Partha Pratim Chakrabarti, and Pallab Dasgupta \\ Indian Institute of Technology Kharagpur, West Bengal, India 721302 \\ \{priyankar, ppchak, pallab\}@cse.iitkgp.ernet.in
}

\begin{abstract}
In this paper we address the problem of generating alternative solutions for implicit AND/OR DAGs which are described using a start state and a set of transformation rules. Unlike the explicit representation of AND/OR graph, for implicitly specified AND/OR graphs, the transformation rules are used to dynamically make a portion of an AND/OR graph explicit as per requirement. We propose an algorithm, ASGAO*, for generating alternative solutions for implicitly specified AND/OR graphs. Experimental results in several domains show that, on an average, our proposed algorithm performs hundred times better with respect to running time and fifty times better with respect to space than the existing algorithm. In view of the renewed research interest towards applying AND/OR graphs in domains like graphical model, constraint satisfaction, and service composition, it is not hard to see useful applications of ASGAO*.
\end{abstract}

\section{Introduction}

Search methods in artificial intelligence are being increasingly used to solve a wide variety of optimization problems from diverse domains like scheduling, routing, constraint satisfaction, CAD for VLSI, etc. In terms of the structure of the state space, OR-graphs, AND/OR graphs and game trees are among the most well studied, among which, AND/OR graphs enable the modeling of alternative choices in conjunctive decompositions of the problem and its subproblems. Recently there has been a renewed research interest towards applying AND/OR structures in a several areas like planning with uncertainty [1], graphical models [2], web service composition [3], etc. In view of these recent research, the problem of generating ordered solutions becomes important for AND/OR graphs. Some of important applications include - (a) developing useful variants of the $\mathrm{AO}^{*}$ algorithm, (b) graphical models, service composition [4], etc. The existing algorithms [54] work on explicit AND/OR graphs - thus the entire search space of the problem has to be made explicit before starting the algorithm. However, making the entire search space explicit is often not required for generating a reasonable number of ordered solutions. For several domains good heuristic functions are available and these estimates can be used to restrict the portion of the search space that is made explicit. We present a new algorithm, ASGAO*, which uses the heuristic estimates to dynamically make the search space explicit like AO* while computing the ordered solutions. Moreover, for problems like matrix chain multiplication, finding secondary structure of RNA, multi-peg tower of Hanoi, etc, whose search spaces have AND/OR structures, the optimal cost of subproblems can be computed efficiently. This exact 
value of the cost information can be used to further reduce the portions of AND/OR graph that is made explicit. Experimental results on domains like, including matrix chain multiplication, secondary structure of RNA, etc., reported in Section 4 , demonstrate hundred times improvement in running time as well as significant improvement in space requirement over the existing algorithm on an average.

\section{Background and Existing Approach}

$G_{\alpha \beta}=\langle V, E\rangle$ denotes an AND/OR directed acyclic graph where $V$ is the set of nodes, and $E$ is the set of edges. The nodes of $G_{\alpha \beta}$ with no successors are called terminal nodes. The non-terminal nodes of $G_{\alpha \beta}$ are of two types - i) OR nodes and ii) AND nodes. The start (or root) node of $G_{\alpha \beta}$ is denoted by $v_{R}$. OR edges and AND edges are the edges that emanate from OR nodes and AND nodes respectively. We use the standard notion of solution graph, $S\left(v_{q}\right)$, rooted at any node $v_{q} \in V$ [6]. By a solution graph $S$ of $G_{\alpha \beta}$ we mean a solution graph with $\operatorname{root} v_{R}$. The notion of cost is as follows. In $G_{\alpha \beta}$, every edge $e_{q r} \in E$ from node $v_{q}$ to node $v_{r}$ has a finite non-negative cost $c_{e}\left(\left\langle v_{q}, v_{r}\right\rangle\right)$ or $c_{e}\left(e_{q r}\right)$. Similarly every node $v_{q}$ has a finite non-negative cost denoted by $c_{v}\left(v_{q}\right)$. We use the notion of additive costs for given solution graph $S$ [6]. For every node $v_{q}$ in $S, C\left(S, v_{q}\right)$ denotes the cost of the solution graph, $S\left(v_{q}\right)$. The cost of a solution $S$ is $C\left(S, v_{R}\right)$ which is also denoted by $C(S)$. We denote the optimal solution below every node $v_{q}$ as $\operatorname{opt}\left(v_{q}\right)$. Therefore, the optimal solution of the entire AND/OR DAG $G_{\alpha \beta}$, denoted by $S_{o p t}$, is opt $\left(v_{R}\right)$. The cost of the optimal solution rooted at every node $v_{q}$ in $G_{\alpha \beta}$ is $C_{o p t}\left(v_{q}\right)$. The cost of the optimal solution $S_{o p t}$ of $G_{\alpha \beta}$ is denoted by $C_{o p t}\left(v_{R}\right)$ or, alternatively, by $C_{o p t}\left(S_{o p t}\right)$. The aggregated cost, $c_{a}$, for an edge $e_{i j}$ from node $v_{i}$ to node $v_{j}$, is defined as : $c_{a}\left(e_{i j}\right)=c_{e}\left(e_{i j}\right)+C_{o p t}\left(v_{j}\right)$

We briefly present the overview of the existing ASG algorithm for AND/OR trees. More details can be found in [4]. ASG algorithm works iteratively - starts by computing the optimal solution and subsequently generates the next best solution over iterations from the solutions that are already generated. In the context of AND/OR trees, $e_{q}$ is used to denote the edge that points to the vertex $v_{q}$. ASG algorithm has been developed on the notion of - (a) marking of an OR edge, (b) swap option and swap operation and (b) the implicit representation of a solution. Consider a solution, $S_{\text {cur }}$, containing an OR edge $e_{i}=\left(v_{q}, v_{i}\right)$, where $S_{c u r}\left(v_{i}\right)$ is actually $S_{o p t}\left(v_{i}\right)$. Now, $e_{i}$ is marked with the cost increment which will be incurred to construct the next best solution from $S_{c u r}$ by choosing another child of $v_{q}$, say $v_{j}$, and this difference is succinctly represented by a swap option $\sigma_{i j}=\left\langle e_{i}, e_{j}, \delta_{i j}\right\rangle$, where $\delta_{i j}=c_{a}\left(e_{j}\right)-c_{a}\left(e_{i}\right)$.

Swap operation basically denotes the application of a swap option to a solution. ASG use a swap option based compact representation, named signature, for storing the solutions. Intuitively, any alternative solution can be described as a set of swap operations performed on the optimal solution $S_{o p t}$. The minimal sequence of swap options corresponding to a solution, $S_{m}$, is defined as the signature, $\operatorname{Sig}\left(S_{m}\right)$, of that solution. The swap list corresponding to a solution $S_{m}, \mathcal{L}\left(S_{m}\right)$, is the list of swap options that are applicable to $S_{m}$. Let $\operatorname{Sig}\left(S_{m}\right)=\left\{\sigma_{1}, \cdots, \sigma_{m}\right\}$ and $\forall i, 1 \leq i \leq m$, each swap option $\sigma_{i}$ belongs to node $v_{p_{i}}$. The application of all other swap options that belong to the OR edges in the path from the root node to $v_{p_{i}}$ is invalidated in the solution $S_{m}$. 
Only the remaining swap options that are not invalidated in $S_{m}$ can be applied to $S_{m}$ for constructing the successor solutions of $S_{m}$. The set of successors of a solution $S_{m}$, denoted by $\operatorname{Succ}\left(S_{m}\right)$, is the set $\left\{S_{m}^{\prime}\right\}$ such that $S_{m}^{\prime}$ can be constructed from $S_{m}$ by applying a swap option in $\mathcal{L}\left(S_{m}\right)$. These concepts are discussed in detail in [4].

ASG algorithm maintains a list, Open, which initially contains only the optimal solution $S_{o p t}$. At any point of time Open contains a set of candidate solutions from which the next best solution in the non-decreasing order of cost is selected. At each iteration the minimum cost solution $\left(S_{\min }\right)$ in Open is removed from Open and added to another list, named, Closed which contains the set of ordered solutions generated so far. Then $\operatorname{Succ}\left(S_{\min }\right)$ is constructed and any successor solution which is not currently present in Open as well as is not already added to Closed is inserted to Open.

\section{Proposed Algorithm for Implicit AND/OR Graphs : ASGAO*}

We use the underlying strategy of node expansion and cost revision of $\mathrm{AO} *$ repetitively in order to make the relevant portion of the AND/OR graph explicit. In the first step, ASGAO* computes the optimal solution using AO*. At this point of time portions of the AND/OR graph have been made partially explicit, i.e., some of the tip nodes of the AND/OR graph are terminal nodes, whereas the others are yet to expand.

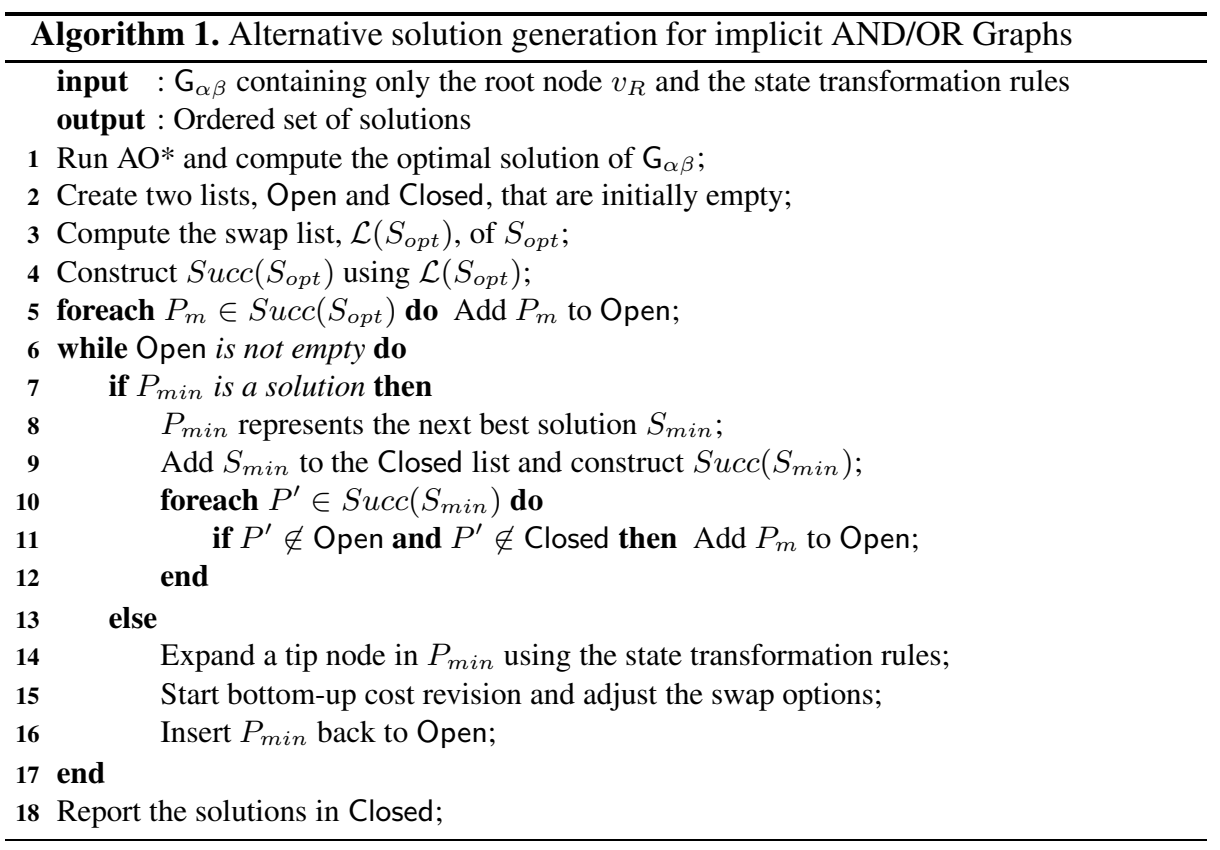

We use $G_{\alpha \beta}$ to denote a partial explicit AND/OR graph which initially consists of the only the root node $v_{R}$. The notion of cost for tip node is as follows. The cost of a tip node is returned by a heuristic function, denoted $h_{\alpha \beta}$. If a tip-node is not a terminal node, $h_{\alpha \beta}$ returns an estimate of the cost of solving the problem represented by that tip-node. On the contrary, when a tip-node is a terminal node, $h_{\alpha \beta}$ returns the cost of 
the atomic subproblem represented by that terminal node. In this paper, we assume that the heuristic function is admissible and consistent. Unlike the ASG, ASGAO* works with potential solution graphs (psgs) [7] of $\mathrm{G}_{\alpha \beta}$. In a potential solution graph, every node with no child is a tip node. Swap options, swap lists, etc. can be computed on the partial explicit AND/OR graphs in likewise manner by considering the heuristic costs of the tip nodes. For partial explicit AND/OR graphs there are two types of swap options. Consider a swap option $\sigma_{p q r}=\left\langle e_{p q}, e_{p r}, \delta_{p q r}\right\rangle$ belonging to node $v_{p}$ whose original edge is $e_{p q}$ and the swapped edge is $e_{p r}$. If both of the optimal potential solution graphs rooted at $v_{q}$ and $v_{r}$, do not have any tip node, then swap option $\sigma_{p q r}$ is a fixed swap option, otherwise $\sigma_{p q r}$ is a changeable swap option.

The first step of ASGAO* is to run $\mathrm{AO}^{*}$ until it generates the optimal solution and after the computation of $S_{o p t}$, the swap options are computed on the partial explicit graph, $\mathrm{G}_{\alpha \beta}$. AO* works over a number of iterations performing (i) computation of the current best psg, and (ii) expansion and cost update, till the optimal solution is found. Then the set of successor psgs of $\operatorname{Succ}\left(S_{\text {opt }}\right)$ are computed and added to Open. It is possible that some of the psgs can actually be a solution depending on whether all the tip nodes are terminal nodes. Next, ASGAO* performs the following over a sequence of iterations. First, the minimum cost psg, $P_{\text {min }}$, is removed from Open and there are the two possibilities. On the one hand, if $P_{\min }$ is a solution, it represents the next best solution $S_{\min }$. ASGAO* adds $S_{\min }$ to the Closed list and constructs the successor set of psgs of $S_{\min }$. Among the successor psgs, each psg which is not currently present in Open as well as is not already added to Closed is inserted to Open. On the other hand, if $P_{\min }$ is not an actual solution, $P_{\min }$ will contain some tip nodes which are not terminal nodes and one of them (chosen arbitrarily), say $v_{t}$, is selected and expanded using state transformation rules. If the cost of $v_{t}$ is changed or its label is changed to solved due to the expansion, a bottom up cost revision step similar to $\mathrm{AO} *$ is triggered. In this cost revision step, apart from revising the cost, the swap options are also updated accordingly. After that, $P_{\text {min }}$ is added back to Open. At any point of time, the Closed list contains the set of ordered solutions generated so far.

Correctness of ASGAO* : We present the key lemma and theorems to establish the correctness of ASGAO* under consistent and admissible heuristics. The details of the proofs are skipped for the sake of brevity.

Property 1. At any point of time during the execution of ASGAO*, the following holds.

1. For every solution $S_{\min }$ present in Closed, $\operatorname{Sig}\left(S_{m}\right)$ contains no changeable swap option;

2. For every psg $P_{m}$ present in Open, at most one changeable swap option can be present in $\operatorname{Sig}\left(P_{m}\right)$;

3. While updating the swap options, the original edge of each changeable swap option which belongs to the signature of a psg, does not change.

Lemma 1. For an initial partial explicit $A N D / O R$ graph $\mathrm{G}_{\alpha \beta}$, for every solution of the corresponding fully explicit graph $\mathrm{G}_{\alpha \beta}^{c}, A S G A O *$ generates that solution.

Lemma 2. ASGSO* adds solutions to Closed in non-decreasing order of cost. 
Table 1. Comparison of time required (in seconds) for AND/OR DAGs corresponding to the search spaces of matrix-chain multiplication with different number of matrices

\begin{tabular}{|c||c|c|c|c||c|c||c|c||c|c|}
\hline \multirow{2}{*}{ \#mat } & \multicolumn{4}{|c||}{ ASG } & \multicolumn{5}{|c|}{ ASGAO* } \\
\cline { 2 - 10 } & $\# n$ & 10 & 20 & 30 & $\# n$ & 10 & $\# n$ & 20 & $\# n$ & 30 \\
\hline 20 & 1521 & 0.026 & 0.029 & 0.032 & 245 & 0.003 & 286 & 0.005 & 314 & 0.006 \\
\hline 30 & 4931 & 0.179 & 0.186 & 0.194 & 506 & 0.006 & 517 & 0.009 & 539 & 0.012 \\
\hline 40 & 11441 & 0.786 & 0.799 & 0.812 & 866 & 0.012 & 879 & 0.016 & 892 & 0.019 \\
\hline 50 & 22051 & 2.590 & 2.612 & 2.637 & 1331 & 0.021 & 1344 & 0.026 & 1357 & 0.031 \\
\hline 60 & 37761 & 6.847 & 6.878 & 6.940 & 1893 & 0.035 & 1900 & 0.041 & 1902 & 0.048 \\
\hline 70 & 59571 & 16.003 & 16.104 & 16.212 & 5765 & 0.234 & 5945 & 0.275 & 5970 & 0.317 \\
\hline 80 & 88481 & 33.968 & 34.187 & 34.408 & 4683 & 0.167 & 6368 & 0.322 & 6404 & 0.384 \\
\hline 90 & 125491 & 66.220 & 66.601 & 66.989 & 5691 & 0.233 & 6915 & 0.380 & 7604 & 0.511 \\
\hline 100 & 171601 & 120.326 & 121.042 & 121.605 & 6656 & 0.300 & 7880 & 0.464 & 8569 & 0.620 \\
\hline
\end{tabular}

Table 2. Comparison of time required (in seconds) for AND/OR DAGs corresponding to the search spaces of secondary structure of RNA

\begin{tabular}{|c||c|c|c|c||c|c||c|c||c|c|}
\hline \multicolumn{1}{|c||}{$\begin{array}{c}\text { Test } \\
\text { Case }\end{array}$} & \multicolumn{4}{c||}{ ASG } & \multicolumn{6}{c|}{ ASGAO* } \\
\cline { 2 - 11 } & $\#$ & 10 & 20 & 30 & $\# n$ & 10 & $\# n$ & 20 & $\# n$ & 30 \\
\hline TC1 & 63237 & 23.999 & 24.022 & 24.127 & 1333 & 0.039 & 1386 & 0.057 & 1476 & 0.089 \\
\hline TC2 & 84274 & 42.919 & 43.289 & 43.253 & 941 & 0.025 & 1399 & 0.058 & 1581 & 0.100 \\
\hline TC3 & 56649 & 19.631 & 19.732 & 19.801 & 1367 & 0.034 & 1682 & 0.054 & 2224 & 0.108 \\
\hline TC4 & 96484 & 53.132 & 53.280 & 53.552 & 1288 & 0.035 & 1408 & 0.054 & 1805 & 0.098 \\
\hline TC5 & 76787 & 33.669 & 33.811 & 34.028 & 1470 & 0.043 & 2296 & 0.085 & 3029 & 0.150 \\
\hline TC6 & 72397 & 31.667 & 31.884 & 32.053 & 1186 & 0.038 & 1927 & 0.074 & 2403 & 0.133 \\
\hline TC7 & 43471 & 10.708 & 10.764 & 10.829 & 789 & 0.019 & 1062 & 0.041 & 1253 & 0.069 \\
\hline TC8 & 49874 & 15.755 & 15.868 & 15.930 & 1055 & 0.030 & 1157 & 0.043 & 1296 & 0.063 \\
\hline TC9 & 45699 & 12.771 & 12.860 & 12.920 & 693 & 0.022 & 854 & 0.041 & 1145 & 0.079 \\
\hline TC10 & 108929 & 70.853 & 71.403 & 74.326 & 1093 & 0.032 & 1409 & 0.059 & 1549 & 0.093 \\
\hline TC11 & 73381 & 31.425 & 31.804 & 32.052 & 1106 & 0.032 & 1152 & 0.051 & 1404 & 0.081 \\
\hline TC12 & 50686 & 15.722 & 15.882 & 16.038 & 1146 & 0.032 & 1426 & 0.062 & 1512 & 0.087 \\
\hline TC13 & 90603 & 50.570 & 50.973 & 51.223 & 1254 & 0.035 & 1453 & 0.054 & 1773 & 0.095 \\
\hline TC14 & 56547 & 18.694 & 18.880 & 19.047 & 1382 & 0.037 & 1605 & 0.057 & 1980 & 0.095 \\
\hline
\end{tabular}

Table 3. Comparison of time required (in seconds) for AND/OR Trees corresponding to the search spaces of 5-peg tower of Hanoi problem with different number of disks

\begin{tabular}{|c||c|c|c|c||c|c||c|c||c|c|}
\hline \multirow{2}{*}{ \#disks } & \multicolumn{4}{|c||}{ ASG } & \multicolumn{5}{|c|}{ ASGAO* } \\
\cline { 2 - 10 } & $\# n$ & 10 & 20 & 30 & $\# n$ & 10 & $\# n$ & 20 & $\# n$ & 30 \\
\hline 8 & 4941 & 0.019 & 0.020 & 0.021 & 101 & 0.004 & 148 & 0.007 & 157 & 0.009 \\
\hline 9 & 17253 & 0.071 & 0.075 & 0.079 & 93 & 0.004 & 130 & 0.005 & 133 & 0.009 \\
\hline 10 & 59049 & 0.227 & 0.246 & 0.261 & 99 & 0.005 & 102 & 0.006 & 197 & 0.010 \\
\hline 11 & 199017 & 0.869 & 1.055 & 1.241 & 145 & 0.006 & 163 & 0.011 & 175 & 0.008 \\
\hline 12 & 662661 & 3.101 & 3.767 & 4.436 & 215 & 0.009 & 288 & 0.016 & 302 & 0.018 \\
\hline 13 & 2184813 & 10.742 & 12.764 & 14.949 & 267 & 0.011 & 410 & 0.018 & 436 & 0.021 \\
\hline
\end{tabular}


Theorem 1. For a given initial partial explicit AND/OR graph $\mathrm{G}_{\alpha \beta}$, every solution of the corresponding fully explicit graph is generated by ASGSO* only once and in the non-decreasing order of costs.

\section{Experimental Results}

To evaluate the performance of the ASGAO*, we have experimented on the following domains - (i) Matrix chain multiplication problem (ii) Secondary structure of RNA (iii) 5-peg tower of Hanoi problem and report the results in Table 1 Table 2 and Table 3 respectively. In these tables, \#n denotes the number of nodes in the AND/OR graph and the time is reported for generating 10,20, and 30 solutions. We also report the number of node present in the explicit graph used by ASG with the number of nodes expanded by ASGAO* for generating same number of ordered solutions. For all of these domains, we compute the exact optimal cost of solving the subproblem represented by any intermediate node using standard dynamic programming approach and use that value as heuristic. Due to the use of exact heuristic, ASGAO* does not invoke the cost revision step and significant improvement is achieved. The details of the test cases used for the secondary structure of RNA as well as the detailed description of each of the domains can be found in [4]. Table 1,3 show that when exact heuristic is used the size of the AND/OR DAG constructed by ASGAO* is significantly smaller than the size of the AND/OR DAG constructed by ASG. For matrix chain multiplication problem, we can observe around hundred times improvement in running time for bigger matrix chains and secondary structure of RNA. For generating 30 ordered solutions, the size of the explicit graphs are around five to twenty-five times larger compared to the size of the corresponding implicit graphs constructed by ASGAO* for matrix chain multiplication and secondary structure of RNA. For tower of Hanoi problem, the improvement is even more significant. Therefore, for domains like constraint satisfaction, planning etc., where good heuristics are available, ASGAO* can be used to generate ordered solutions efficiently, thereby opening up interesting research directions.

\section{References}

1. Russell, S., Norvig, P.: Planning. In: Artificial Intelligence: A Modern Approach, 2nd edn., pp. 375-461. Prentice-Hall, Englewood Cliffs (2003)

2. Dechter, R., Mateescu, R.: AND/OR search spaces for graphical models. Artif. Intell. 171(2-3), 73-106 (2007)

3. Lang, Q.A., Su, Y.: AND/OR graph and search algorithm for discovering composite web services. International Journal of Web Services Research 2(4), 46-64 (2005)

4. Ghosh, P., Sharma, A., Chakrabarti, P.P., Dasgupta, P.: Algorithms for generating ordered solutions for explicit AND/OR structures. J. Artif. Intell. Res (JAIR) 44, 275-333 (2012)

5. Elliott, P.: Extracting the k best solutions from a valued AND/OR acyclic graph. Master's thesis, Massachusetts Institute of Technology (2007)

6. Martelli, A., Montanari, U.: Additive AND/OR graphs. In: Proceedings of the 3rd International Joint Conference on Artificial Intelligence, Morgan Kaufmann Publishers Inc., San Francisco (1973)

7. Dasgupta, P., Chakrabarti, P.P., DeSarkar, S.C.: Multiobjective heuristic search in AND/OR graphs. Journal of Algorithms 20(2), 282-311 (1996) 